\title{
LA GIUDAD Y EL MEDIO AMBIENTE
}

\author{
VALENTÍN IBARRA \\ Sergio Puente \\ Martha SCHTEINGaRT
}

\section{INTRODUCCIÓN}

El PRESENTE TRABAJO ES SÍNTESIS de la versión preliminar del marco conceptual del proyecto Ecoville. ${ }^{1}$ Su principal objetivo es la definición de los parámetros teóricos y analíticos, dentro de los cuales debería enmarcarse no sólo la interacción, sino también la integración y posterior fusión de dos distintas áreas de conocimiento: la ecología y el desarrollo urbano. No se pretende, de ninguna manera, consumar una teorización globalizante; señalar los distintos niveles en los que debería darse la conceptualización, destacar los tópicos centrales a abordar y definir la complejidad de tal intento rebasaría con mucho los alcances de este trabajo.

1 El proyecto Ecoville se propone estudiar, en forma comparativa, el impacto del desarrollo de las grandes ciudades sobre el medio ambiente. En él partiçipan equipos de investigación de distintos países de América Latina, Asia y África, bajo la coordinación general del Center for Environmental Studies de Toronto y el auspicio de la International Federation of Institutes of Advanced Study (IFIAS). Este marco conceptual orientará los estudios que se realicen en la región latinoamericana, los cuales estarán referidos a los casos de São Paulo, México, Santiago, Caracas, Bogotá y Quito. 
El estudio de dicha interacción no se deriva exclusivamente de una válida inquietud académica. Es resultado de una paulatina toma de conciencia y preocupación social ante la manifestación y consolidación de problemas que, no siendo exclusivos de la ciudad, son más evidentes en la cotidianidad citadina. En efecto, el acelerado proceso de urbanización, orientado principalmente a las grandes concentraciones urbanas, que han experimentado los países en vía de desarrollo se ha visto acompañado, casi siempre, por un creciente deterioro del medio ambiente y de la calidad de vida.

Por la visión holística que la caracteriza, la ecología sería el área de conocimiento pertinente donde encontrarían coherencia los esfuerzos aislados por resolver los problemas del medio ambiente: $\mathrm{Si}$ bien la ecología delinea los parámetros de un marco de normatividad global, susceptible de modelar una relación armónica sociedad-naturaleza y adecuada principalmente a los complejos ritmos reproductivos de los ecosistemas, no ha definido una estrategia social de apropiación de la naturaleza bajo las presentes condiciones de desarrollo social. Tal vez ello se deba a que esta definición rebasaría su propio campo de acción. Esto es evidente al tratarse de fenómenos estrictamente sociales, y en particular de lo urbano. De ahí que cualquier intento por conocer el impacto que sobre el medio ambiente tiene el crecimiento de las ciudades, exige la explicación previa de la modalidad teórica que adoptaría la interacción de dos distintas áreas del conocimiento: el desarrollo urbano y la ecologia.

De esta forma, este trabajo se estructura en tres apartados. En el primero se intenta definir los espacios de dicha interacción, principalmente en la ciudad o lo urbano, bajo la perspectiva de la determinación histórica de la relación sociedad naturaleza. En el segundo, luego de presentar de manera general la problemática urbana tal como se da en las sociedades capitalistas, se procede a incorporar su dimensión ambiental considerando la lógica de producción de su base material. Y, finalmente se analizan las funciones urbanas básicas en su relación con el medio ambiente.

\section{UN INTENTO DE INTEGRACIÓN DE LA DIMENSIÓN DEL MEDIO AMBIENTE EN EL ANÁLISIS DE LO URBANO}

No obstante el renovado interes por comprender la lógica y definir los factores determinantes de la organización y funcionamiento del 
espacio urbano-regional, la complejidad del fenómeno ha dificultado hasta ahora la formulacion de una conceptualización integrada.

A esta inquietud científica se ha sumado una creciente preocupación social por los problemas que resulten o se manifiesten mâs nítidamente en las concentraciones urbanas. Dentro de éstos, el deterioro de la naturaleza ha adquirido particular importancia. Los esfuerzos realizados para entender sus implicaciones y adoptar medidas correctivas han sido igualmente limitados y fragmentados; principalmente porque su análisis y comprensión están condicionados por la integración teórico-metodológica de la dimensión ambiental, al análisis de los procesos de estructuración social del espacio.

Al hablar de "integración" se alude a la complejidad del intento por "fusionar" dos áreas de conocimiento con bases teóricas propias y con distintas legalidades analíticas: ${ }^{2}$ el campo de la ecología y del desarrollo urbano-regional. Intento de mayor dificultad, porque la "fusión" exigiría la formalización de una nueva área de conocimiento con distinta especificidad teórica (Leff E., 1980).

La relación sociedad-naturaleza, la forma como el hombre se apropia e interactúa con ella para asegurar su sobrevivencia y reproducción, es el eje analítico que permitiría delinear con mayor rigor la modalidad de dicha integracion. ${ }^{3}$

De esta forma, una primera aproximación a una conceptualización integrada debería de manejarse en tres niveles: integración enmarcada en la legalidad analítica de las ciencias sociales para los problemas propios de la reproducción social en su conjunto particularmente, para los relativos a la producción y organización del espacio urbano; integración con referencia a la legalidad analítica de las

2 Por "área de conocimiento" entendemos el intento científico por conocer los orígenes y procesos determinantes de un fenómeno social o natural reconocido como un "campo de problemas". En este intento pueden confluir una o varias disciplinas. Las áreas de conocimiento tienen distintos grados de desarrollo en su proceso de consolidación como ciencia.

3 Para los propósitos que nos ocupan, centraremos nuestro análisis en el concepto de apropiación, sin menoscabo de otras modalidades que dicha relación pudiese adoptar. Concebimos el concepto de apropiación como la acción unilateral del hombre de apoderarse, de servirse de la naturaleza, para satisfacer sus necesidades, sin más razón que su voluntad. Este acto se inscribe dentro de un sistema valorativo de la naturaleza social, e históricamente determinado en función de la especificidad cultural, organización social y grado de conocimiento de la naturaleza por parte de la comunidad que se la apropia. Sería otro el concepto si el hombre normase su relación con la naturaleza en consideración a sus efectos reactivos, minimizando no sólo los negativos o degenerativos para él como especie, sino también los que lo son para la naturaleza. 
ciencias naturales al tratarse de las alteraciones de los ecosistemas; y por último, definición de las mediaciones conceptuales que articularían ambos ámbitos en consideración de los pasajes por sus correspondientes legalidades, si lo central son los efectos biunívocos y la determinación de los parámetros de lo que sería una modalidad orgánica y equilibrada de esta relación.

La pertinencia de tal conceptualización no estaría garantizada por una simple aproximación interdisciplinaria, donde cada doctrina reclamase su participación según su cuerpo teórico y en función de los distintos enfoques contenidos en él. Dependería principalmente de que los recortes teóricos sean adecuados a la complejidad de las distintas instancias del fenómeno. Se requeriría igualmente indicar sus articulaciones y los momentos del análisis en los que cada disciplina pudiese operar con cierta autonomía sobre determinados aspectos del fenómeno (Adorno W. T., 1978).

Con cuerpos teóricos propios, el desarrollo urbano regional y la ecología han avanzado en forma independiente. En el curso de su desarrollo sus problemáticas han ido convergiendo, principalmente a partir del momento en que el desarrollo urbano y regional incorpora al medio ambiente, admitiendo que la reproducción de las ciudades y sus regiones presupone un proceso de apropiación y transformación de la naturaleza.

Son varios los intentos hechos para romper con aproximaciones unilaterales del problema, a través de transposiciones de conceptos o categorías de una disciplina a otra. Dentro de ellas, la más significativa es el ecosistema para conceptualizar el fenómeno social de lo urbano; la ciudad concebida como ecosistema en función de las interrelaciones entre los organismos vivientes, su medio ambiente abiótico y sus flujos de energía. Esta visión se ha ampliado incorporando a las variables bióticas y abióticas variables sociales (político-culturales y del comportamiento) (Douglas I., 1982). Si bien el concepto de ecosistema presupone una aproximación globalizante su empleo ha sido variado, divergente y fragmentado.

Se ha hecho igualmente énfasis en distintos aspectos del problema; particularmente en la elaboración de analogías: la ciudad como proceso de concentración de población se ha explicado de acuerdo con una visión ecológica, presuponiendo que su organización y funcionamiento se da de manera similar a la adoptada por otras especies naturales, ignorando asi la importancia determinante de los procesos sociales, sustantivos a la organización de la ciudad.

No obstante, en estos esfuerzos siempre han quedado pendientes 
la conceptualización de los procesos sustantivos, las mediaciones analíticas para articular ambos campos de estudio y los momentos en que éstas se inscriben en sus correspondientes legalidades. La ausencia de estos aspectos es lo que justifica la elaboración de un marco conceptual que sirva de base a todo intento por comprender la relación proceso de urbanización-medio ambiente.

Para tal propósito, se impone como exigencia preliminar una estricta demarcación del uso apropiado de los conceptos centrales de ambas áreas de conocimiento. Dos conceptos afloran como los objetos de estudio de las áreas del conocimiento del desarrollo urbanoregional y de la ecología, respectivamente: el modo de producción (principalmente como modalidad de apropiación del espacio naturaleza) y el ecosistema. Nos centraremos en este último debido a la laxitud con la que ha sido utilizado y porque nos permite precisar la correspondencia y divergencias entre ecosistemas y medio ambiente $y$ al mismo tiempo destacar la importancia del hombre en la transformación de la naturaleza.

\section{Correspondencias y divergencias entre ecosistema $y$ medio ambiente}

Desde la óptica ecológica, el hombre establece intercambios con la naturaleza como una especie más dentro de las otras que la integran. La modalidad con que se efectúa este intercambio y sus efectos en la reproducción de cada una de las especies y del medio ambiente que integran constituyen su objeto teórico (Odum, 1977).

Este medio ambiente se supone como un todo organizado, pero diferenciado - como una discontinuidad de la continuidad que nos ofrece la naturaleza en su totalidad - en conjuntos discretos de especies animales, vegetales y de microorganismos que coexisten en forma estable con los otros elementos físicos, químicos y geológicos. Dichos organismos discretos, que se presentan como las últimas unidades articuladas en que puede ser dividida la naturaleza, al tener la capacidad de autorregularse, de permanecer en equilibrio dinámico, de reproducirse por sí mismas, y de regenerarse en caso de alteraciones por algún fenómeno externo, constituyen para la ecología unidades ambientales que pueden ser tipificadas como ecosistemas. La importancia de esta concepción estriba en que inscribe al hombre como "una especie más" dentro de un todo organizado, resaltando la 
IBARRA/PUENTE/SCHTEINGART: CIUDAD Y MEDIO AMBIENTE 115

modalidad de su interacción en la reproducción y estabilidad del ecosistema.

La correspondencia entre medio ambiente y ecosistema pierde consistencia en la medida en que el hombre incrementa su apropiación de la naturaleza, en función exclusivamente de las exigencias intrínsecas a la lógica de su organización social y no de las leyes de homeostasis, dinámica propia del ecosistema. Al perder sus cualidades distintivas (principalmente las de reproducción y autorregulación) el medio ambiente del cual depende el hombre dejaría de ser en rigor un ecosistema.

De ahí la importancia de conocer la lógica y evolución con que el hombre se apropia la naturaleza. Y para ello habría que remitirse a otra legalidad analítica: el modo de producción adoptado históricamente por el hombre para asegurar su reproducción como entidad social.

Ha habido cambios en los modos de producción que han normado históricamente la interacción del hombre con la naturaleza. Igualmente cambiantes son los ecosistemas de los que forma parte.

Se pueden distinguir dos formas fundamentales de apropiación de los ecosistemas por parte del hombre.

- Cuando la naturaleza, en tanto eçosistema, se presenta al hombre como un recurso renovable en sí mismo, con capacidad de reproducirse en forma independiente de su acción.

- Cuando la naturaleza, para asegurar su resiliencia y reproducción, requeriría de la mediación de una cantidad de trabajo por parte del hombre para convertirse en un artificio ecológico. Al perder su capacidad de autorreproducción se consideraría un recurso no renovable.

En este último caso, al mediar el hombre en su reproducción, la naturaleza estaría fungiendo ya como un medio de trabajo y no como objeto de trabajo, como lo sería en el primer caso: la tierra deviene el medio para producir los frutos que anteriormente la naturaleza le brindaba al hombre en forma espontánea.

El desarrollo del hombre ha hecho que la mayor parte de los ecosistemas con los que interactúa dejan de serlo o se conviertan gradualmente en artificios ecológicos.

En este proceso de transformación, podrían distinguirse tres instancias con las que el hombre se relaciona diferencialmente (Toledo, 
1981): el medio ambiente natural, el medio ambiente transformado y el medio ambiente social.

El medio ambiente natural (MAN) sería con el que el hombre establece una relación de apropiación, respetando sus leyes de autorreproducción y resiliencia, adoptando una modalidad de intercambio ecológico.

El medio ambiente transformado (MAT) serían los espacios naturales que han sufrido una mediación de trabajo por el hombre. No se utilizan como objeto de trabajo sino como medio de trabajo (principalmente la tierra, cuyo producto ya no sería el resultado espontáneo de las leyes que rigen el ecosistema, sino que estaría determinado por la acción deliberada del hombre: fase de la naturaleza domesticada, cultivada). Serían medios naturales que han transferido a una instancia exterior sus cualidades sustantivas de autorreproducción y resiliencia dejando propiamente de ser ecosistema.

El medio ambiente social (MAS) o artificial se define por la especificidad espacio-temporal del intercambio económico entre distintos conglomerados de unidades productivas. Este puede ir desde un nivel local o regional hasta uno nacional o internacional. En el MAS se rompe la autosuficiencia del intercambio ecológico, que establece el hombre con una sola unidad ambiental o ecosistema para asegurar su reproducción.

A diferencia de los otros medios ambientes, el MAS se sitúa totalmente dentro de la legalidad analítica de lo social. E1 hombre dejaría de interactuar como especie, tal como lo considera el cuerpo teórico de la ecología, para interactuar como ente social a través del intercambio económico, al que antecede, en casi todos los casos, la realización de un intercambio ecológico: intercambio de energía en los distintos niveles tróficos. Siendo varias las unidades productivas que intercambian, varios y distintos son los medios ambientes y ecosistemas apropiados. Puede plantearse, así, que todo intercambio económico tiene en su base un intercambio ecológico. Es la lógica del primero que determina la forma cómo el hombre se inscribe en el segundo. Mas para comprender sus efectos sobre el funcionamiento del ecosistema es necesario remitir el análisis, con cierta autonomía, a la legalidad de lo ecológico.

La atención analítica en el MAS se centraría en la forma de organización social de la producción: participación socialmente diferenciada en el nivel de la producción, distribución, circulación y consumo del producto natural apropiado. Históricamente definido, y por lo tanto cambiante, determina a su vez la modalidad tecnológica 
y el grado de apropiación de los ecosistemas. De esta forma, no es homogéneo su grado de conservación, transformación o degradación resultante de la acción del hombre.

\section{Lo urbano como medio ambiente socio-artificial y no como ecosistema}

En la medida en que el hombre, socialmente organizado, incrementa históricamente sus intercambios se produce una transformación cualitativa del medio ambiente: pasaje de lo natural y transformado a lo social.

Si no restringimos el concepto de medio ambiente social al tipo y nivel de intercambio, o a la modalidad tecnológica utilizada, y tomamos en consideración el grado de transformación del medio ambiente, o de su artificialidad lograda por la acción del hombre, podremos introducir un aspecto distintivo, propio a nuestro objeto de estudio: lo urbano, o la ciudad.

Desde esta óptica, lo urbano se nos presenta como la condensación material de trabajo social acumulado históricamente, resultante de un proceso de máxima apropiación y transformación de la naturaleza. Su principal característica es la reducción de la variedad biótica del medio ambiente natural que le sirve de soporte, y la supresión de las principales funciones que permitirían caracterizarlo como ecosistema: la autorreproducción y la resiliencia. Éstas serían transferidas a la mediación del hombre.

Al estudiar el impacto del proceso de producción de la base material y del funcionamiento de los asentamientos humanos sobre el medio ambiente, estamos obligados a inscribirnos dentro de la lógica de la legalidad social que lo norma, pues la artificialidad sobre la naturaleza en lo urbano sólo se entiende a partir de dicha legalidad.

Tal intento debería de partir de la dependencia de la ciudad de otros ámbitos para garantizar su reproducción. En efecto, la división social del espacio entre campo y ciudad, sujeta a esta última a una dependencia con la otra instancia espacial para garantizar su reproducción, principalmente en cuanto a materias primas, alimentos y energía. Son excepcionales los casos en que un centro urbano es autosuficiente (el grado de artificialidad que lo caracteriza no se lo permitiría); pero puede serlo de su medio ambiente natural o trans formado inmediato: su entorno natural. El mayor o menor grado de transformación depende de distintos procesos de apropiación, de variados y distantes medios ambientes naturales (ecosistemas) o trans- 
formados. El medio ambiente inmediato de la ciudad "tendrá una mayor o menor participación en dicha reproducción, pero en la medida que el centro urbano se desarolla, incrementa su dependencia a 'medios ambientes alejados" "(Gutman, 1980). La complejidad e intensidad de la dependencia entre medios ambientes naturales $y / 0$ transformados y la ciudad puede transitar entre medios ambientes sociales artificiales.

Las consideraciones anteriores nos permiten inferir dos instancias de análisis en la evaluación del impacto ambiental del proceso de urbanización.

- La relativa al "medio ambiente inmediato", base material del proceso de producción del marco construido urbano. Es propiamente su medio ambiente circundante. Un medio ambiente transformado, que al incorporarse al urbano, deja de ser medio ambiente inmediato para ser medio ambiente social-artificial, como lo es cualquier ciudad. Este análisis se inscribiría dentro de la legalidad de lo social. Tomaría como referente algunos elementos de la biosfera (agua y aire principalmente) puesto que no se puede observar de acuerdo a las leyes que regulan un ecosistema.

- La instancia relativa al o a los "medios ambientes alejados". De acuerdo con el grado de alteracion podrían conceptualizarse como ecosistemas y su análisis se encuadraría dentro de la legalidad de lo ecológico, o como medios ambientes sociales artificiales y en este caso se estudiaría en la legalidad de lo social. Es claro que al tratarse de medidas correctivas de apropiación por el hombre, se volvería a tomar como referencia la legalidad de lo social: cambios de tecnología en el tipo de producto cultivado, en la intensidad de su explotación, etc. Al evaluar el impacto en el ecosistema de dichos cambios habría una confluencia de legalidades, mas no una fusión.

La división conceptual entre medios ambientes inmediatos y alejados se fundamenta en términos de distancia, pero son igualmente importantes los aspectos relativos al tiempo, o a lo que podríamos llamar acceso físico-material y acceso social. La proximidad espacial no garantiza un fácil acceso: ciertas condiciones geológicas, edafológicas, topográficas, etc., pueden influir para que otro medio ambiente, aunque más distante, sea más accesible en términos de 
tiempo. De igual importancia son los factores sociales que determinan los circuitos de circulación de los productos y energía, destinados a la ciudad: trátese de la modalidad de su comercialización (terciarización o monopolización), que no obstante la cercanía puede hacer inaccesible el producto a la ciudad o de innovaciones tecnológicas en la proporción o en los medios de transporte, lo que permite iniciar la apropiación de un medio ambiente.

De lo hasta aquí expuesto, se deriva que hay dos niveles de caracterización del "medio ambiente": la "transformación y artificialidad" que ha hecho el hombre de la naturaleza siendo la intensidad y cualidad de los "intercambios, ecológicos y económicos", factores constitutivos y el espacio-naturaleza que sirve de soporte a la ciudad la versión más acabada de artificialidad; y de la "accesibilidad espacial y social" de la ciudad para apropiarse los distintos medios ambientes (naturales y transformados, ecosistemas o no), indispensables para garantizar su reproducción.

Cabe señalar que los procesos de utilizacion de la naturaleza no son los únicos que impactarían el medio ambiente. Existe un reflujo de residuos, resultantes de la producción y el consumo urbanos, que igualmente los alteraría, afectando principalmente al propio ambiente urbano. Lo urbano no puede concebirse exclusivamente como importador de energía o expulsor de desechos, connotativo de un sometimiento de explotación o expoliación de otros medios ambientes. Al concebirse el consumo productivo de lo urbano como proceso de cosificación de energía y materias primas en bienes producidos y al ser éstos posteriormente consumidos por otros ámbitos espaciales, se puede hablar de una exportación de energía cosificada por parte de lo urbano a los ambitos originarios o a otros: maquinaria e instrumentos pertenecientes al sector primario.

\section{LO URBANO Y EL MEDIO AMBIENTE}

\section{Aspectos generales}

Hemos realizado la diferenciación entre medio ambiente natural, transformado y social artificial, ubicando a la ciudad dentro de este último. Suficiente en una primera instancia para referirnos a la relación hombre-naturaleza. Es necesario, ahora definir los procesos sociales urbanos, para adentrarnos en un análisis más profundo de su relación con los medios ambientes, cuya unidad debe ser destacada en su recomposición dentro del conjunto de la sociedad. 
Después de presentar una definición general de lo urbano, tomaremos cada uno de los elementos que hemos considerado básicos y analizaremos su conexión con los medios ambientes: alejados, inmediato e interno.

Existen diferentes definiciones de lo urbano, de acuerdo con la orientación teórica adoptada que relevarán diversos aspectos del fenómeno a estudiar y llevarán al planteamiento de diferentes tipos de relaciones.

Desde nuestro punto de vista, y continuando con lo expuesto, es necesario definir los elementos básicos y los procesos sociales en forma conjunta para el caso de las ciudades capitalistas, haciendo referencia después a las especificidades detectadas en los centros urbanos de los paises subdesarrollados, como los latinoamericanos.

En este contexto, la ciudad es el medio socio-espacial en el que las actividades ahí asentadas, y el modo específico en que se aglomeran los elementos que las constituyen, reproduce la vida material del hombre y relaciones, inherentes a ese tipo de sociedades. En este marco se inscriben las actividades de producción, consumo, intercambio y gestión, base de la reproducción social. Los procesos sociales no podrían entenderse sin un análisis del sector productivo, de las respuestas de los sectores populares urbanos y de la acción del Estado.

Por otra parte, los procesos económicos y sociales -incluidos en la producción, el consumo y el intercambio- tienen una especialidad concreta; requieren de soportes materiales que se organizarán en el territorio de acuerdo a sus requerimientos locacionales, mediados por la lógica de producción del espacio urbano. Cada actividad tiene como base un objeto inmobiliario, que circula en forma independiente del otro; ellos constituyen en conjunto el "valor de uso complejo" de la ciudad, ${ }^{4}$ resultante de la articulación de valores de uso individuales. Algunos de estos objetos son mercancías, producidas por el capital privado o el Estado, mientras otros lo son, por los sectores populares, sólo como valores de uso (aunque luego puedan entrar a la circulación). Existen diferentes formas de producción de esos objetos, en los que están implicados distintos agentes, soportes de relaciones sociales, cuyas modalidades específicas están regidas por la división técnica y social del trabajo (Topalov, 1972).

4 EJ sistema espacial que constituye la ciudad es un valor de uso específico, diferenciado del valor de uso de cada una de sus partes, consideradas separadamente; es un valor de uso complejo que nace de la articulación en el espacio de valores de uso elementales (Topalov, 1979). 
La renta del suelo urbano (como parte de las sobreganancias originadas en el proceso de producción de los objetos inmobiliarios y en las actividades generadoras de ganancias que se desarrollan en su interior y que se embolsa el propietario del suelo) es un componente fundamental de la producción del marco construido, pues constituye un mecanismo de asignación de las diferentes actividades urbanas y de los grupos sociales en el territorio. Influye en la determinación de la división funcional y social del espacio y en la configuración desigual del mismo (Lipietz, 1974).

La división social del espacio no se presenta como “. . la proyección directa del sistema de estratificación social, sino como un efecto de la distribucion del producto entre los sujetos y del producto vivienda en el espacio, así como de la correspondencia entre estos dos sistemas de distribución. . . ello exige deducir la composición del espacio a partir del estudio de su proceso de producción. . ."(Castells, 1972).

La distribución de las actividades productivas, de consumo e intercambio, resultaría de la combinación de las necesidades particulares de localización de cada una de ellas (que veremos más adelante), de la lógica de producción de los objetos inmobiliarios que les sirven de base material y de la estructura general de usos del suelo en la ciudad, ya determinada en gran medida por la renta del suelo y heredada además de épocas históricas anteriores.

La vivienda es el elemento urbano que ocupa una mayor proporción del espacio de la ciudad, de la expansión de la mancha urbana y de la mencionada división social del espacio. Como objeto inmobiliario, su producción está vinculada al suelo; así la renta afecta su localización y constituye una limitacion más al acceso de la mayoría de la población. Al problema con la tierra deben sumarse otros, provenientes de las especificidades del proceso productivo mismo, como los largos períodos de rotación del capital industrial involucrado y de circulación, que limitan la producción del sector privado y hacen que ella sólo resulte rentable cuando se dirige a los estratos solventes de la población. La intervención del Estado, sobre todo a través del financiamiento, se hace necesaria para permitir que aquellos que no tienen acceso al mercado privado, puedan pagar en forma escalonada y en relación a sus ingresos. Coexisten también diversas formas de producción de la vivienda: la producción por encargo, la promocional privada y pública y la autoconstrucción; ellas colaboran en la generación de espacios urbanos para diferentes estratos sociales (Schteingart, 1979). 
En cada ciudad se presenta una combinación particular de formas de producción-circulación de los objetos inmobiliarios y en especial de la vivienda. En países subdesarrollados, y particularmente en América Latina, se han observado formas no mercantiles en una medida mucho mayor que en los paises desarrollados; grandes contrastes entre modos avanzados y primitivos de producción y crecientes dificultades para que el Estado pueda participar activamente en la producción de los bienes indispensables que afectan el consumo de las mayorías urbanas. Esto agudiza la organizacion desigual del espacio y las grandes carencias y déficits para los sectores pobres urbanos. ${ }^{5}$

\section{Ciudad y medio ambiente}

A los procesos físicos y químicos de transformación de elementos materiales que conciernen a la producción y que forman parte de un circuito primario de materia (natural y modificada) donde se incluye el intercambio, se les suma la actividad también transformadora del consumo.

Así, junto a los determinantes sociales de la producción, el consumo y el intercambio, se encuentran los mecanismos que hacen que la materia prima, extraída de fuentes bióticas y abióticas, se transfigure a lo largo de varias etapas.

Los procesos de producción, consumo e intercambio implican una apropiación de la naturaleza, a través de la utilización de materias primas y productos intermedios que afectan medios ambientes más o menos alejados de la ciudad. Al mismo tiempo, generan residuos y deshechos (gaseosos, sólidos, húmedos); algunos afectan la calidad del aire, el agua y el suelo de la ciudad y de su entorno inmediato.

También la producción de objetos inmobiliarios, soporte de las actividades mencionadas, afectan el medio ambiente. La construcción requiere de insumos que provienen de medios ambientes más o menos

5 En el caso de la vivienda se ha podido comprobar, por un lado, la penetración creciente de empresas promotoras privadas y públicas en la producción destinada a los estratos de la demanda solvente, y por otro, la utilización generalizada de una forma primitiva como la autoconstrucción; si bien ella se vuelve dominante para los grupos pobres urbanos comienza últimamente a resultar también inaccesible para amplias capas de la población. Los recursos de los organismos estatales de vivienda son cada vez más limitados para hacer frente a las necesidades de las mayorias y sus condiciones habitacionales se deterioran ostensiblemente (Jaramillo, Schteingart, 1983). 
naturales y alejados; no genera quizás tantos residuos como las industrias transformadoras de materiales y convertidoras de energía, pero tiene una influencia decisiva en la determinación del medio ambiente urbano. De esta manera la producción del marco construido urbano, sin dejar de afectar medio-ambientes alejados, determina el medio ambiente de la ciudad y su entorno inmediato.

Las formas que asumirá la destrucción y reemplazo del medio ambiente natural o transformado (bosques, zonas cultivadas, etc.), por el medio construido dependerá del ritmo de crecimiento de la población urbana, de la lógica de producción del marco construido (que ya mencionamos) y de la forma de ocupación del suelo (construcción de viaductos, crecimiento a saltos del tejido urbano, construcción de vivienda individual o colectiva, etc.).

Las tecnologías aplicadas en las formas de producción del marco construido, implican la utilización de materiales precarios o poco elaborados (como en la autoconstrucción o en la pequeña vivienda individual producida por encargo) o el uso de elementos industrializados y de técnicas modernas de construcción (como en los edificios públicos, comerciales, o en la vivienda colectiva o en serie realizada a través de la producción promocional más avanzada). Esas tecnologías producen diferentes impactos en el medio ambiente inmediato de la ciudad o en medios ambientes más alejados, de acuerdo con el grado de elaboración de los insumos utilizados y con las mediaciones en la apropiación de elementos naturales (no es lo mismo construir con madera, ladrillo y embarro que con acero y cristal).

La ciudad es, entonces, desde el punto de vista de su interacción con el medio ambiente: importadora de energia (articulándose con medio-ambientes más o menos naturales y alejados); transformadora de la misma para ser exportada o consumida localmente, en forma cosificada, utilizadora del medio natural inmediato (que sirve de soporte físico a la producción del marco construido); y el lugar donde se evacúan residuos de distinto tipo (que afectan el aire, el agua y la tierra de la ciudad y su entorno).

Cuando hacemos referencia al medio ambiente de la ciudad podríamos, a nuestro criterio, considerar dos aspectos del mismo:

El medio ambiente construido urbano, resultado contradictorio de la producción y circulación de cada uno de los valores de uso individuales, generados de acuerdo al principio de la ganancia, mediante la apropiación privada del suelo y en el contexto de una estructura social, en la que se da un desigual acceso de diferentes estratos de la población al consumo urbano. Una de las principales manifestaciones 
de esa forma de producción del marco construido es la división funcional y social del espacio (separación en zonas industriales, comerciales y habitacionales para diversos sectores sociales) y las densidades de ocupación del mismo (relación entre espacios ocupados por edificios de diferentes alturas o infraestructuras y los espacios libres o verdes) y la disposición de los elementos del marco construido.

El medio ambiente físico-natural urbano, referido a sus elementos más "naturales", como el aire, el agua y la tierra y al impacto que tienen sobre los mismos residuos y deshechos. En la determinación de este medio-ambiente, el contex to geográfico urbano juega un papel significativo: la altitud, la topografía y el clima afectan el impacto que los residuos pueden tener sobre el aire, el agua y la tierra.

El medio ambiente urbano, estaría condicionado por los elementos constitutivos de los dos aspectos considerados. Creemos que el primer aspecto (además de su importancia intrínseca para observar los niveles de consumo de la población) es relevante para los fines de este trabajo porque afecta directamente al segundo. Por ejemplo, las densidades, la altura de los edificios, o la presencia de espacios verdes, pueden afectar la calidad del aire, y agudizar la contaminación proveniente de procesos productivos o de intercambio. Por otra parte, la contaminación del aire puede afectar la existencia de áreas verdes de la ciudad, al incidir sobre las posibilidades de crecimiento de algunas especies vegetales.

Pero lo más importante es que a través de ellas puede explicarse la distribución desigual de la energía (principalmente alimentos, electricidad, gas y carburantes) y de los efectos contaminantes de las actividades urbanas. Se piensa que existe una estrecha vinculación entre la división social y funcional del espacio, y las formas en que se distribuye el consumo energético y la contaminación ambiental. Así se configurarían los contextos socioambientales intraurbanos.

Aunque en todas las ciudades existen esos contextos socioambientales, en los países subdesarrollados se dan en forma más contrastada y son expresión de una extrema división social del espacio, donde se produce una polarización en la distribución de los beneficios y de los medios de consumo urbano. Contrastan así, aquellos donde se asien. tan estratos de la población con muy reducido poder adquisitivo, bajos niveles educativos y de salud, viviendas precarias y sin servicios, escaso consumo energético y altos niveles de contaminación con otros donde los sectores más pudientes disfrutan de lujosas viviendas, espacios verdes y buena calidad del aire y agua. Esto no significa que no existan ciertos espacios urbanos (las áreas centrales, sobre todo) 
donde los problemas ambientales afectan por igual a todos los estratos sociales.

Es necesario aclarar también que las diferencias socioespaciales no sólo se dan a nivel intraurbano. Hemos puesto énfasis en las de este nivel por la índole del trabajo desarrollado, pero también se dan en otros medio ambientes.

LOS PROCESOS DE PRODUCCIÓN, CONSUMO E INTERCAMBIO: SU BASE MATERIAL Y SU RELACIÓN CON EL MEDIO AMBIENTE

\section{La producción industrial}

La producción industrial constituye el elemento motor de la ciudad, la base para la organización del espacio urbano y es a partir del desarrollo de las "condiciones generales" de esa producción que se puede explicar la concentración urbana y las modalidades de su organización interna (Lojkine, 1978). Sin embargo, esto no significa que toda la ciudad se fundamente en la industria; es necesario tomar en cuenta las interacciones entre la producción y los demás elementos básicos de la ciudad (el intercambio, el consumo).

- En una sociedad concreta, en una determinada ciudad, coexisten diversas formas de producción, varias etapas de la división capitalista del trabajo (desde la cooperacion simple hasta la automatización), así como relaciones de producción precapitalistas (la artesanía) (Topalov, 1979), que requieren de diferentes medios de producción y fuerza de trabajo (más o menos calificada), tendrán necesidades de localización variables dentro del espacio urbano, se relacionarán de manera distinta con el intercambio y el consumo y afectarán diferencialmente los ecosistemas y el medio ambiente.

Así, en las etapas más avanzadas de la división social del trabajo, parece existir una tendencia acentuada a la eliminación de las barreras geográficas, que antes interferían en los planes de localización industrial. Se puede observar una homogeneización del espacio en cuanto a la provisión de energía, al reemplazar el carbón por la electricidad y al densificar la red de distribución energética. Asimismo, al intensificarse el uso de sintéticos, un conjunto importante de ramas industriales deja de orientar su localización hacia el lugar de extracción de materias primas naturales. Y, finalmente, la revolución tecnológica de los transportes y comunicaciones, debilita el rígido emplazamiento que otras industrias mantenían por su dependencia con respecto a ciertos mercados. 
Sin embargo, esta propensión a la desconcentración espacial, que ya se manifiesta con claridad en los paises avanzados no es perceptible todavía en los países subdesarrollados. Por el contrario, el proceso concentrador de la industria en pocos puntos del territorio nacional mantiene su inercia pese a las consecuencias sociales negativas que de ella resultan (Garza, 1981).

Comúnmente esta situación se asocia al papel que le corresponde jugar a cada país en la división internacional del trabajo y a las condiciones materiales de la producción, que los mismos estados construyen para apoyar el crecimiento económico. De este modo, el examen de situaciones concretas ha mostrado la permanencia de economías para el sector industrial en los países subdesarrollados, lo que permite suponer que los costos crecientes de urbanización, creados por la concentración industrial, no se reparten equitativamente entre los sectores de la población. En efecto, se presenta una apropiación desigual de los beneficios económicos de la aglomeración que no tiene correlación con el pago de los costos.

Uno de los costos sociales más palpables, originados por la concentración industrial, es el de la contaminación ambiental. Empíricamente se han identificado las fuentes más contaminantes del aire y del agua y se ha estudiado su impacto en el contorno de la ciudad bajo determinadas condiciones geográficas y climáticas. Éste es un nivel válido de análisis pero insuficiente si no se enmarca en el proceso de trabajo, perteneciente a aquellas industrias reconocidas como contaminadoras.

Recuérdese, en primer lugar, que cada rama industrial requiere de materias primas de distinta índole. Esto nos remite al impacto sobre otros ambientes del lugar donde se implantó la industria. Así, en razón del carácter de la materia prima, al extraerse y procesarse previamente puede registrarse un deterioro relativo de medios ambientes alejados (naturales, transformados $u$ otros medios ambientes urbanos). Conviene insistir en esta cuestión porque no necesariamente la industria, cuyo proceso de transformación contamina el ambiente urbano donde se localiza, es la misma que afecta medios ambientes alejados al obtener sus materias primas. Ahora, la gama de impactos ambientales puede ser muy variada y combinarse con efectos sociales y económicos, de manera que su jerarquización conjunta es distinta, según el país de que se trate.

Además de las materias primas básicas que entran en la composición del producto acabado, se precisa de insumos adicionales inclui dos los medios energéticos. Como en el caso de las primeras, la extrac- 
ción y procesamiento, anteriores a su aprovechamiento, en un proceso industrial determinado, nos conduce a la evaluación de las consecuencias ecológicas que resultan de ellas. De nueva cuenta, lo más probable es que el ámbito de análisis trascienda al medio ambiente urbano.

Conviene acotar que no existe una correspondencia permanente válida entre el producto elaborado por una industria y los insumos requeridos. En este sentido, es pertinente un seguimiento de los cambios tecnológicos más relevantes de las diversas ramas industriales en el medio ambiente (sustitución gradual o inmediata de materias primas naturales por sintéticas o reconversión energética). Consecuentemente, en la base de ese rastreo histórico no deben estar ausentes las transformaciones económicas nacionales y mundiales que condicionan tales cambios.

Entrelazado con el punto anterior se encuentra el proceso más directamente relacionado con el medio ambiente urbano: el funcionamiento de todos los mecanismos que dan lugar a las transformaciones físicas y químicas que permiten obtener un proceso acabado. Pero en la misma medida en que se constituye en un elemento central para comprender integralmente el vinculo industria-medio ambiente, en los países subdesarrollados es el menos conocido en su funcionamiento interior. Se registran irregularmente estimaciones sobre cada tipo de contaminante industrial, se estima el posible daño ecológico que provocan aisladamente o mezclados y transfigurados por la acción atmosférica, etc., gracias a los avances de la investigación ecológica en los países desarrollados; sin embargo, debido al ritmo del desarrollo tecnológico industrial en el que prácticamente no se participa y que se incorpora ciegamente en contextos geográficos distintos al de su origen, aunado al nulo interés por conocer los posibles efectos perniciosos que sobre la salud acarrean tanto el proceso técnico de transformación como los materiales utilizados, es más difícil alcanzar un conocimiento pleno del proceso de trabajo. Por otra parte, todo avance tecnológico incorporado a la industria urbana exige cambios en las técnicas de extracción de materias primas (explotación intensiva de suelos, por ejemplo). Por tanto, la separación analítica del proceso de trabajo en la industria conviene enmarcarla en una visión global y dinámica, pues de lo contrario la política ambiental, derivada de tal análisis, tendría un alcance espacial y temporal limitado.

En síntesis, éstos son los componentes que conforman el proceso de trabajo que debería especificarse aún más para hacerlo útil a la investigación concreta. Tal especificación sólo podría provenir de la misma evidencia empírica, que ayudaría a formular hipótesis inter- 
medias. Con este propósito, y sin pretender ser exhaustivos, se enuncian a continuación algunas proposiciones en las que se intenta eliminar supuestos generalizadores implícitos en lo expuesto hasta aquí:

- No existe una adscripción tecnológica unívoca a cada industria. Una rama industrial puede incluir diversas tecnologías y, por lo tanto, diferentes impactos ambientales.

- Existe un movimiento espacial continuo de la industria dentro de la ciudad. No es casual que una empresa con una determinada tecnología se localice en un lugar determinado.

- Existen ramas industriales cuya tecnología demanda un tamaño de planta, que determina el volumen de desechos sólidos, líquidos o gaseosos potencialmente contaminantes.

- Una planta industrial aislada puede generar materias contaminantes fácilmente asimilables por el medio ambiente sin causar perjuicios a la naturaleza y al hombre. Sin embargo, un conjunto de plantas de diversas industrias es capaz de ocasionar un deterioro significativo del medio ambiente. En este sentido resulta pertinente hablar de un umbral ecológico, estrechamente conectado no sólo con el tamaño de la ciudad sino con el tipo, número y tamaño de plantas localizadas en la misma.

- Los ámbitos de influencia, en términos del grado de contaminación, son variados; de manera general se hizo alusión a ellos (medios ambientes alejados, contextos socioambientales intraurbanos, etc.). No obstante, hasta ahora ha permanecido ausente un ámbito conectado directamente a la actividad industrial: el medio ambiente de la fábrica. Tiene importancia incluirlo puesto que la contaminación hacia el exterior de la planta puede ser menor si se compara con lo que sucede internamente, es decir, con el daño que sufren los trabajadores.

Todos estos aspectos deben tenerse en cuenta en cualquier política ambientalista. Sin embargo, desde un punto de vista normativo riguroso es insuficiente conocer qué tipo de tecnología produce cuáles y cuántos contaminantes. Esto supondría la posibilidad de seleccionar tecnologías, menos perjudiciales al medio ambiente y más 
acordes con la reproducción continua de la naturaleza, lo cual, al margen de la organización socioeconómica de cualquier país sería un objetivo ilusorio. La evidencia histórica ha mostrado la presencia de una estrecha interdependencia entre el sistema de producción y la lógica y evolución del avance tecnológico (Sagasti, 1981), por lo que pretender cambiar el signo de esa lógica exógenamente al sistema productivo sería un camino frustrado.

Tal atadura es más aguda en los países subdesarrollados. En las ciudades de América Latina, por ejemplo, la producción industrial está particularmente dominada por la penetración del capital internacional; éste, al mismo tiempo que trasfiere el uso de tecnologías avanzadas a empresas nacionales, exporta procesos de fabricación altamente contaminantes. Obviamente, esto restringe la cobertura y eficacia de las políticas nacionales de conservación y mejoramiento del medio ambiente. Sin embargo, no por ello es factible afirmar que los estados nacionales carecen de márgenes de maniobra aprovechables en beneficio de la población, sobre todo teniendo en cuenta que una proporción importante de la economía se encuentra regulada por el Estado.

Si por uso racional de la tecnología en la industria privada entendemos la adecuación de los instrumentos producidos por la ciencia y la técnica para los fines de la industria, es decir a la obtención de ganancias dentro de unos límites institucionales que la protegen y promueven, la industria paraestatal, sin ser ajena totalmente a ese principio racional, tiene la exigencia de internalizar normas sociales que condicionan su funcionamiento (Habermas, 1981). Esto no significa que el Estado invente su propia tecnología, con arreglo a un sistema de valores sociales entre los que se encuentra el respeto a la naturaleza y mucho menos en el caso de los países subdesarrollados. Sin embargo, el objetivo de armonizar entre todos los sectores sociales y la prevalencia de axiomas, admitidos y consolidados históricamente (pero reinterpretados y refuncionalizados), conduce al Estado a moderar la exacción de la naturaleza por la industria.

Las vías que el Estado se puede formular con el propósito de regular y evitar los daños causados a la naturaleza por la tecnología están en la misma acción en la industria paraestatal y la planificación que incluye todas las leyes, reglamentos, organismos y planes creados para tal efecto.

Las restricciones técnicas y sociales impuestas a ambas vías para los países subdesarrollados han quedado indicadas. No obstante, conviene subrayar las más importantes: 
- El grado de dependencia tecnológica de los países subdesarrollados obliga a la importación de equipos anticontaminantes. La falta de un dominio sobre los procesos industriales contribuye al desconocimiento de la técnica anticontaminante. Este hecho explica la poca importancia que se le atribuye a la contaminacion, en relación al conjunto de problemas sociales que se padecen: agregar equipo anticontaminante de procedencia extranjera significa una pérdida de rentabilidad; es común que las industrias privadas ignoren toda reglamentación al respecto. El Estado, por su parte, que dispone de ciertas industrias estratégicas, puntales de la base económica, consideraría un lujo distraer recursos para fines no prioritarios.

- La elección racional entre alternativas de acción que supone la planificación requiere no sólo del conocimiento de las reglas y procedimientos técnicos en que descansa toda acción (incluido el proceso técnico de la planificación) sino de un conocimiento aproximado de los procesos económicos, sociales y políticos que conforman una sociedad determinada. La adquisicion de habilidades y destrezas respecto a las reglas técnicas de acción es una prolongación del desarrollo científico y tecnológico, aplicado a la industria, pero por sí mismas no resuelven los problemas, sobre todo si se desconocen los procesos de interacción social. La dificultad en los países subdesarrollados para normar la conservación del medio ambiente tiene que ver con los dos aspectos: el fracaso en la estrategia para resolver problemas relativos a la instrumentación de la planificación y de la tecnología industrial, y la desvinculación con todo análisis socioeconómico (Kade, G., 1970).

- Las "perversiones", creadas por el mismo crecimiento económico, encuentran menos obstáculos en los países subdesarrollados que en los altamente industrializados. A las condiciones particulares de la acumulación de capital y a los límites estructurales de la intervención estatal, se suma un factor que añade otro rasgo esencial a la caracterización aqui presentada. La gran oferta de mano de obra en relación a la capacidad de absorción por la industria, la presencia de un alto nivel de desempleo, la existencia de una masa marginal de subempleados y los bajos niveles 
salariales, influyen en la estructuración del esquema de reivindicaciones sociales y en la modalidad de las presiones sociales. Así, la demanda para mejorar las condiciones de vida está más relacionada con la supervivencia humana que con el cuestionamiento del problema ambiental urbano.

Pero, al lado de estas limitaciones, los fenómenos económicos de la industria pueden facilitar un mayor control de los procesos de trabajo generadores del deterioro ambiental. A pesar de que se confirma empíricamente una dispersión en el espacio urbano de las etapas del proceso de trabajo correspondiente a una misma rama industrial, económicamente se observa una mayor centralización del capital; esto es, grandes corporaciones no sólo tienden a ser prominentes en una rama sino que integran horizontalmente todos aquellos sectores industriales, comerciales, etc., que son soporte de la actividad industrial básica. La unificación del interés económico, que abarca desde la extracción de la materia prima hasta la distribución del producto final, permite una intervención estatal con criterios globales y sin la necesidad de negociar políticamente con una diversidad de intereses conflictivos.

\section{El consumo urbano}

Conviene aclarar que el consumo urbano no constituye un rereptáculo pasivo de la producción industrial; tampoco se concibe como instancia autónoma y con un peso específico que per se puede explicar el vínculo entre lo urbano y el medio ambiente. Consideramos que el consumo está sujeto a múltiples determinaciones entre las que destaca la producción industrial; pero ésta no excluye la acción que el mismo proceso del consumo social ejerce sobre la continuidad de ésta ni los factores socioculturales que influyen en la composición y evolución de los patrones de consumo.

Por otra parte, el consumo es el eje en torno del cual gira la reproducción material de toda sociedad. Sin embargo, este hecho debe mostrarse detalladamente para hacerlo útil a nuestros propósitos de la investigación proyectada.

Frecuentemente, el consumo en la ciudad se asocia con la reproducción de la fuerza de trabajo; no obstante, considerarlo así limita su función a un sector de la población (los asalariados) que, aunque mayoritario, no agota todo el espectro englobado. Por ello, resulta 
conveniente recordar que la ciudad es la configuración espacial, resultante de la estructura social dominante y de los elementos socioespaciales, heredados de estructuras sociales anteriores. Esta cuestión es de particular importancia para entender el modo en que se distribuyen los objetos de consumo entre los diferentes grupos sociales, incluido aquel que sirve de asiento fundamental al consumo urbano: la vivienda. Es inevitable hacer referencia al consumo urbano como un proceso social de reproducción diferencial, de acuerdo con los grupos sociales existentes (definidos en términos de su inserción en la economía de la ciudad), proceso que encuentra su manifestación espacial, si tomamos como referencia la vivienda, en la segregación social, asimilable al concepto de contextos socioambientales intra urbanos, cuando se hacen explícitos los aspectos ambientales.

Al margen de las características sociales del proceso de consumo, éste tiene un contenido material. Así, no todo lo que se adquiere en el mercado es consumible ni está dispuesto para su aprovechamiento inmediato. Se precisa eliminarles lo accesorio e inútil (empaques, envolturas, etc.) o someterlos a una transformacion ulterior (alimentos, por ejemplo). Otros objetos cuyo uso es prolongado obligan a su mantenimiento y/o limpieza; operaciones que exigen de más insumos, aparatos, equipo e instalaciones así como de un sitio donde llevarlas a cabo.

Aunque no todo el consumo se verifica en la vivienda, una gran parte de los productos finales de la industria privada encuentran su destino precisamente ahí. Esto permite continuar con la dirección metodológica de la sección anterior, en la medida en que los bienes de consumo final son susceptibles de clasificarse, conforme a la desagregación realizada para la reproducción industrial. ${ }^{6}$

Esta clasificación que nos proporciona todo el espectro de bienes consumidos (manejables empíricamente), junto con los procesos de transformación ocurridos en la unidad doméstica, permiten reconstruir las interrelaciones básicas entre el consumo y el medio ambiente.

Partiendo de la clasificación de bienes es posible mostrar el primer vínculo material entre la praducción y el consumo, teniendo como denominador común al medio ambiente. De la contrastación entre el deterioro parcial del medio ambiente, adjudicable a cada rama

6 Conviene hacer una salvedad. Una gran cantidad de productos de consumo final también son empleados en las actividades productivas, comerciales y de servicios. Sin embargo, es de suponer que tales productos son consumidos en su mayor parte por las familias. 
industrial (en cualquiera de los niveles del proceso de trabajo), y el reconocimiento social de sus productos (qué tipo de necesidades sociales cubre) es plausible derivar criterios para delinear estrategias de acción. Una política de descentralización industrial incorporaría criterios económico-ambientales, reforzados con principios basados en las necesidades sociales en contraposición de intereses particulares. ${ }^{7}$

Una gran proporción de las mercancías adquiridas para el consumo, debido a sus características físicas por un lado y a la posibilidad de ofrecerlas competitivamente por otro, obligan a darles una determinada presentación exterior no consumible en sí misma. Esta presentación es producida por un trabajo independiente del que se requiere para elaborar el producto que contiene, pero en el campo del consumo se muestra como unidad indisoluble. $Y$ es precisamente en el consumo donde se manifiestan las consecuencias que sobre la salud y el medio ambiente genera el material usado en la fabricación del bien y su recipiente. Ilustraciones de ello son los desperdicios domésticos de envolturas, cajas, etc., que no pueden degradarse biológicamente o que alteran al t.iismo bien que supuestamente conservan en buen estado.

La finalización real de los bienes, a semejanza de la producción industrial, significa una ulterior transmutación de la materia. De este proceso resultan subproductos, algunos de los cuales son reciclables; otros, simplemente se suman a los desechos producidos por el metabolismo económico de la ciudad. Por otra parte, dicho proceso tiene lugar en la vivienda.

En la vivienda se ha incorporado el equipamiento necesario para permitir la entrada de los insumos requeridos en la efectivización del consumo, así como el equipamiento necesario para dar salida a los desechos derivados del consumo. De la eficacia de estos mecanismos depende en gran parte el mejoramiento de las condiciones de vida de la población. Primeramente, porque el aprovisionamiento oportuno y suficiente de agua, energia, etc., incide sobre los niveles de bienestar social. En segundo lugar, porque la falta de previsión en los sistemas utilizados para abastecer de insumos y evacuar desechos puede: $a$ ) agotar las fuentes de recursos no renovables; $b$ ) afectar medio-ambientes alejados cuando la extracción irracional de recursos naturales hace disminuir su productividad; c) causar daños irreversibles en medios

7 Esto hace alusión a otra clase de consecuencias que no se observan en el medio ambiente pero que se localizan en la esfera del consumo y repercuten en las condiciones de vida. Es decir, alimentos no nutritivos, artículos ajenos totalmente al cuadro de necesidades de la mayoría de la población, etc. 
ambientes naturales o transformados por el inadecuado traslado de los residuos domésticos.

La gravedad del impacto ambiental y las repercusiones socioeconómicas está asociada con la escasa o nula precaución de los organismos públicos, responsables de los equipamientos colectivos; las dimensiones y las condiciones geográfico-climáticas de la ciudad, el tamaño de su población y la forma en que ésta se distribuye en el espacio urbano contribuyen a acentuar los problemas del medio ambiente.

Es posible inferir de lo expresado hasta ahora, la subordinación que guarda el consumo con respecto a la producción desde el punto de vista material. Las transformaciones del objeto consumido están determinadas por los elementos físico-químicos que lo componen; un envase de lenta degradación ocasiona indefectiblemente un deterioro ambiental. Por otra parte, la diferenciación social en la ciudad se manifiesta también en la distribución desigual de los equipamientos de la vivienda, lo que provoca que las mismas actividades de reproducción alteren el medio ambiente urbano. En otras palabras, la intensidad del problema ecológico no sólo se relaciona con qué se consume sino con quién y cómo lo consume.

Pero no sólo existe un condicionamiento objetivo de carácter material-económico; también encontramos un espacio dentro del consumo social que varía en función de la historia cultural de cada sociedad, y se refiere a las prácticas cotidianas de los residuos domésticos. Éste es el punto donde confluye el debate sobre la "conciencia ecológica" de la que parecen carecer los pobladores de los países subdesarrollados.

A reserva de que este aspecto sea enriquecido con una investigación comparativa, conviene formular una hipótesis muy general que indique los parámetros en los que se enmarcan las pautas de comportamiento ecológico en los países subdesarrollados: la posibilidad de construir una conciencia ecológica depende de las características sociales y políticas del país. Específicamente, el nivel del desarrollo económico y el grado en que ha sido superado el problema de la supervivencia de la mayor parte de la población, condiciona la aparición y evolución del interés social por el medio ambiente. Sin embargo, se presentan factores restrictivos nacidos en la interdependencia de toda índole entre países; existe una determinada permeabilidad cultural de modo que ciertos patrones culturales pertenecientes a países desarrollados han sido paulatinamente internalizados en los países no industrializados; por otra parte, no obstante la intención de difundir ampliamente como principio axiológico el respeto por la 
naturaleza, la percepción social del problema ambiental y la preocupación por resolverlo es tan desigual como la distribución de la riqueza en un país.

Así, pues, dado el grado de desarrollo alcanzado y el inequitativo reparto de la riqueza, el Estado enfrentará serios obstáculos para promover la mencionada conciencia ecológica. Es decir, en la medida en que existan metas sociales prioritarias relacionadas con carencias acuciantes en las condiciones de vida inmediatas, el mejoramiento ambiental tenderá a subestimarse ideológicamente. No obstante al Estado le compete la acción directa a través de la construcción de los equipamientos colectivos y de la prestación de los "servicios públicos", con lo que influye directamente sobre las condiciones ambientales de la ciudad.

En primer término cabe mencionar al conjunto de equipamientos que apoyan la producción de los servicios destinados al mantenimiento $\mathrm{y}$ ampliación de las capacidades intelectuales, así como a la reproducción de las relaciones sociales predominantes (servicios educativos y culturales, por ejemplo). El análisis de la construcción de los equipamientos (escuelas, teatros, etc.), se inscribe evidentemente en la producción del marco construido urbano, mientras que la producción del servicio que ocurre simultáneamente con el consumo, carece de incidencia sobre el medio ambiente. La única relación que es posible destacar se refiere a los sistemas de educación que integran, en los contenidos formativos, nociones sobre la relación entre el hombre y la naturaleza. Los alcances realistas de este objetivo ya se indicaron en párrafos anteriores.

En segundo lugar, se incluyen los equipamientos, ligados a la vivienda que ya fueron tratados anteriormente. Como se observaba, éstos son imprescindibles para disfrutar de los bienes comprados de la industria, y su aprovisionamiento eficaz puede significar menores impactos sobre el medio ambiente.

Finalmente, la última categoría englobaría al resto de los servicios públicos. Las deficiencias en la calidad del consumo familiar, la falta de conciencia ecológica de la población y los desperfectos en la transformación de agua potable obligan a dedicar un gasto público creciente para solventar los servicios públicos de salud, de limpieza y de disposición de residuos. Estos servicios actúan como indicadores de las carencias en el consumo social, y las deficiencias en el abastecimiento de los demás servicios públicos. En la medida en que disminuyan las enfermedades gastrointestinales y del sistema pulmonar (características de los países subdesarrollados), que exista un menor 
contenido de materia orgánica en los desperdicios domiciliarios, que se registren menores índices de fugas de agua potable, etc., se conocerá el éxito de cualquier política ambiental urbana.

\section{El intercambio}

El crecimiento económico en casi todos los países ha desembocado en una distribución desigual de las actividades económicas en pocos puntos del territorio y en una expansión física cada vez más compleja de algunas ciudades. Estos dos procesos han exigido también la ampliación y modernización de los medios de circulación social y material: la transformación de la planta productiva en cada sociedad (innovaciones tecnológicas incorporadas, crecimiento del tamaño de nuevas ramas industriales por el desmembramiento de otras, etc.) y la inexistencia de una regulación espacial que permita acoplar las unidades de producción, de distribución, gestión y consumo a los componentes internos de cada una de ellas, han conducido al perfec cionamiento y especialización de las redes de intermediación comercial y financiera (circulación social) y a la revolución de-los sistemas de transporte y comunicación (circulación material).

En el ámbito estrictamente urbano, la localización de las unidades de producción, distribución y consumo, obedeciendo a su propia lógica de funcionamiento y a condiciones específicas que privan en cada ciudad según el país de que se trate (organización política del territorio nacional, factores ideológico-culturales, formas especiales heredadas del pasado y renta del suelo urbano), da lugar a formas que dificultan el flujo de los intercambios urbanos entre cada una de las unidades. Esta circunstancia requiere de un sistema de transporte y comunicaciones que elimine las fricciones del espacio a través del tiempo, es decir, que facilite la accesibilidad urbana. Por otro lado, las nuevas vías de comunicación y construcción de transporte, indisolublemente ligadas a los fines de especulación del suelo urbano o de rentabilidad de !a industria de la construcción, van determinando y modificando la estructura preexistente al incorporar más espacio.

Sin embargo, aun cuando se derive de lo anteriormente señalado la presencia de un condicionamiento mutuo entre la división funcional del espacio urbano y los medios de circulación material, la primera constituye el factor dominante de esa relación (Topalov, 1979).

Los elementos que conforman el intercambio (medios de circulación social) tienen también su base material y forman parte del marco 
urbano junto a las unidades de producción, de gestión y de consumo. Por otro lado, al concebir la instancia socioespacial es conveniente, para los propósitos de enfatizar el vínculo entre lo urbano y el medio ambiente, reducirnos al examen del funcionamiento del transporte urbano como mecanismo mediador del intercambio físico en la ciudad. Además, en la medida en que las actividades comerciales no son transformadoras, no obstante que requieren de energía y equipo en su funcionamiento, no se consideran determinantes esenciales del impacto ambiental en la ciudad.

El transporte urbano facilita el tráfico de mercancías y personas. Al traslado de mercancias se le considera una prolongación del proceso de producción industrial y guarda una estrecha dependencia con las transformaciones de la producción industrial. Concretamente el transporte permite el intercambio físico de mercancías entre los distintos componentes del espacio urbano.

En cuanto al traslado de personas, los viajes más importantes son los que se realizan entre el hogar y el lugar de trabajo; se incluyen, por tanto, los viajes personales efectuados a las unidades de producción, distribución y gestión. Dada la necesidad de disponer oportunamente en la planta industrial de materias primas, instrumentos y fuerza de trabajo, el papel que juega el transporte, al trasladar lo que se considera las condiciones básicas de la producción, es económicamente más importante que el traslado de personas por motivos distintos del trabajo. Estos últimos están más relacionados con la reproducción de los distintos sectores sociales (viajes a centros de educación, viajes de recreo, etcétera).

Cuando se hace referencia al sistema de transporte urbano, consideramos como parte integrante de él todos los componentes requeridos para su funcionamiento. La incorporación del estudio sobre cada uno de los medios de transporte y la tecnología que les corresponde es de suma importancia para evaluar los impactos ambientales; si descuidamos el análisis de la forma en que se organiza socialmente la producción, reduciríamos la causa del problema ambiental a orígenes puramente tecnológicos.

Es difícil elaborar un esquema de análisis válido para todos los países subdesarrollados, que permita integrar la diversidad de agentes sociales que intervienen en los distintos niveles de la producción y gestión del transporte. Tal vez, el rasgo común para todas las sociedades es la intervención de organismos públicos en la construcción de la "infraestructura vial", lo cual se explica por las dificultades que 
enfrenta el empresario individual para desarrollar esta actividad de manera rentable.

En las principales ciudades de América Latina encontramos diversas formas de organización del servicio de transporte de personas: empresas privadas de autobuses; empresas estatales de autobuses, tranvías y trolebuses, transporte subterráneo eléctrico estatal; empresas cooperativas de "microbuses"; empresas familiares; etc., reflejo del proceso modernizador en el transporte y del nivel económico del país. En cambio, en el transporte de mercancías, predomina la pre sencia polarizada del estado y de empresas privadas de transporte.

Por otro lado, desde el punto de vista técnico, se han desarrollado modos de transporte, de acuerdo al objeto transportado. Para el transporte de personas se ha consolidado uno de ellos: el automóvil privado. La necesidad de crear ciertas condiciones generales a la producción, que deja en segundo término el apoyo al transporte de personas, y la acción particular de la industria automotriz ha conducido al fortalecimiento de éste dentro del sistema de transporte de personas y, como veremos, al grado de contaminación del medio ambiente urbano.

Finalmente, es pertinente resumir las grandes invariantes de diferente orden que sirven de marco al desarrollo del transporte en los países subdesarrollados; sin ellas, el estudio del transporte sobre el medio ambiente resultaría incompleto:

- El crecimiento económico dependiente limita las posibilidades financieras y la innovación tecnológica autónoma. La vulnerabilidad de estos países en las crisis económicas debilita aún más sus planes y programas económicos y científicos de largo plazo (si existen) estrechando las alternativas de solución a las necesidades crecientes de transporte.

- La dependencia tecnológica, en materia de transporte, condiciona el uso de determinados materiales y combustibles, cuyo aprovisionamiento puede significar una mayor dependencia y un mayor impacto ambiental.

- La estructura urbana en transformación y crecimiento continuo (división social y funcional del espacio) es generadora de nuevas necesidades de transporte.

El transporte se caracteriza por ser uno de los mayores consumidores de energía, situación que se asocia con el crecimiento econó- 
mico. En sí mismo, el transporte urbano de personas se ha mostrado como un consumidor voraz al basarse en forma progresiva en el uso del automóvil y al ser éste el medio de traslado más ineficiente en términos de combustible gastado por pasajeros transportados y distancia recorrida (Murthy, 1978).

La cuestión anterior no tendría mayor relevancia si la fuente de esa energía fuese renovable, pero ésta se extrae de combustibles fósiles, cuya dotación se encuentra en cantidades limitadas.

Dada la dependencia extrema que mantiene el transporte con el combustible y derivados del petróleo, no es de extrañar el grave deterioro de los medios ambientes naturales, cercanos a los campos de explotación y a los centros de refinación del petróleo. Los estudios sobre la destrucción de la flora y la fauna por la extracción del petróleo empiezan a ser más conocidos, aunque a la vez más ignorados, por los responsables de la política petrolera.

A los problemas económicos y ambientales, suscitados por la explotación petrolera, es necesario agregar los efectos ecológicos, inherentes al funcionamiento de los sistemas predominantes de transporte urbano basados en los vehículos automotores. La contaminación del aire (dependiendo de la difusión de los contaminantes en áreas más o menos alejadas), que resulta del proceso de combustión que hace mover a los vehículos, parece tener efectos más reducidos sobre los medios ambientes naturales circundantes que sobre la propia población urbana, situación distinta a lo que ocurre con el drenaje de los residuos y desechos provenientes de las unidades de consumo (la vivienda). En este sentido, el impacto del transporte sobre el medio ambiente puede observarse a través de los cambios del microclima urbano, condicionados por la morfología, el clima, la altitud, la dirección de los vientos, etc.; igualmente, la proliferación de particulas sólidas, de monóxido de carbono y de otros compuestos en el aire, puede tener efectos nocivos sobre la salud de los habitantes de la ciudad, que se detectarían a través de un registro de la clase y volumen de contaminantes y de los cambios ocurridos en las tasas de mortalidad y morbilidad, según las zonas de la ciudad. Una molestia adicional es el ruido, producido por la excesiva cantidad de vehículos en circulación.

No debe olvidarse que, al margen de los impactos de los vehículos automotores sobre el medio ambiente físico, el transporte de personas se distribuye diferencialmente entre sectores de la población urbana. El transporte actúa como mecanismo discriminatorio en el medio ambiente social, guardando una correspondencia con la manera en que los impactos sobre el medio ambiente físico recaen sobre la 
población (se ha descubierto, por ejemplo, que el contenido de plomo en la sangre de las personas varía conforme al lugar de residencia, el cual depende, invariablemente, de la solvencia económica de los residentes) (Kapp, 1976).

Algunos de los problemas ambientales, originados por el transporte, hace pensar en la conveniencia de una intervención planificadora que elimine o reduzca el daño ecológico. Las medidas que se recomiendan para incluir en todo plan, se basan más en el sentido común que en un análisis riguroso. Por ejemplo, reorganizar el sistema de transporte en el que se privilegie el transporte masivo ahorrador de combustibles, la utilización de energéticos no fósiles, la desconcentración industrial, etc., son políticas que requiren grandes cambios en la sociedad y que sólo en el largo plazo puede acarrear resultados exitosos.

\section{CONCLUSIÓN}

El marco conceptual que hemos presentado revela el análisis de los procesos sociales urbanos, a diferencia de otros enfoques que han tratado la relación urbanización-medio ambiente, considerando la ciudad como una caja negra en la que entran insumos de ecosistemas exteriores, y se transforman en el interior de la misma, generando impactos a los ecosistemas que se dan dentro y fuera del medio urbano.

El enfoque aquí propuesto no se propone minimizar los aspectos ecológicos del fenómeno considerado, "sociologizando" el medio ambiente, sino que intenta ubicar adecuadamente los factores ecológicos que juegan en la relación sociedad urbana-medio ambiente.

Para ello hemos considerado fundamental explicar en qué consiste lo urbano; cuáles son las actividades básicas que lo definen, la dinámica del funcionamiento de las mismas y las implicaciones que tiene en la configuración de su base material, regida por la lógica de producción del marco urbano.

Dentro de este intento de ubicar jerarquizadamente los distintos elementos y relaciones que componen lo urbano, van surgiendo conceptos, categorías de análisis y relaciones, sin las cuales sería imposible entender las causas de los problemas ambientales.

Las necesidades de insumos, sus formas de distribución, la generación de desechos y su impacto ambiental, no resultan de procesos ciegos sino precisos y vinculados al funcionamiento de las ciudades y 
a la existencia de un sistema de agentes sociales actuantes, que se describen y explican en sus componentes y condiciones fundamentales.

Estamos conscientes de que este marco conceptual puede resultar un poco esquemático, ya que no se han incorporado suficientemente algunos aspectos de tipo social y cultural que dificultan aún más este complicado y poco desarrollado campo de análisis. Sin embargo, creemos que en este caso una simplificación de esa realidad ha sido necesaria, al presentar un nuevo esquema de análisis -contrapuesto a otros muy difundidos- que intenta relevar aspectos frecuentemente ignorados o minimizados.

También se ha querido abundar más en ciertas descripciones de los procesos implícitos en la producción industrial, el consumo, el intercambio y en la producción de la base material de la ciudad (para luego ver sus relaciones con el medio-ambiente); desarrollar con detalle los aspectos técnicos de la contaminación o la destrucción del medio ambiente natural, temas mucho más trabajados y especificados en la literatura especializada.

Este marco conceptual, que servirá de base para estudiar el impacto del desarrollo metropolitano en el medio-ambiente en los países subdesarrollados, ha tomado elementos teóricos de análisis referidos a las ciudades capitalistas, tratando de poner énfasis en problemas y aspectos específicos del capitalismo periférico, donde los procesos urbanos adquiren rasgos particulares, que afectan el medio ambiente con un mayor grado de intensidad.

Por último, la utilización del marco presentado implicaría el aporte de diferentes disciplinas tanto de las ciencias sociales, como de las ciencias físicas y naturales. Pero ello no significa necesariamente que cada línea de investigación se desarrolle dentro de un área disciplinaria. Por el contrario, es conveniente que las diversas disciplinas converjan al análisis y explicación de áreas problemáticas que resulten de los planteamientos básicos del marco. Por ejemplo, el estudio de cada una de las actividades urbanas y de la lógica de producción de su base material, vinculadas a sus necesidades de insumos e impactos ambientales, debe recurrir tanto a la economía y sociología urbanas, como a los estudios ecológicos y de integración científica que el esquema propuesto ha intentado tratar.

Asimismo, las diferencias socioespaciales intraurbanas y la generación de contextos socioambientales, merecen la consideración de una gama muy amplia de variables y, una compleja red de relaciones donde también podrían converger diversas disciplinas.

A partir de los elementos conceptuales expuestos en este trabajo 
se podría instrumentar la utilización de métodos y técnicas de análisis complejo, sin excluir la posibilidad del uso adecuado de modelos conceptuales y formalizaciones matemáticas. Sin embargo, consideramos necesario aclarar que ese uso sólo sería pertinente si a través del mismo no se produce una simplificación inaceptable de los procesos y fenómenos apuntados que los reduzca a simples expresiones que poco tienen que ver con la realidad que pretenden representar.

\section{Bibliografía}

Adorno, W. T., "Sobre la lógica de las ciencias sociales", La Lógica de las Ciencias Sociales, Textos Vivos, núm. 6, México, Grijalbo, 1978.

Barrera, C., Diagnóstico ambiental urbano, Bogotá, Oficina de Investigaciones Socioeconómicas y legales, OFISEL, 1980.

- Sistema urbano y medio ambiente, ibid., 1980.

Castells, M., La cuestión urbana, México, Siglo XXI, 1972.

DANSEREAU, P., Man-environment interaction at the settlement level, Vancouver, United Nations Conference on Human Settlements, 1976. 1981.

Douglas, I., "The city as an ecosystem", documento presentado en el seminario Urbanización y medio ambiente en los paises subdesarrollados, México, El Colegio de México, marzo, 1982.

Emmanuel, A., L'echange inégal, París, Maspero, 1969.

GARZA, G., "El proceso de industrialización de la Ciudad de México: 1845-2000", Lecturas del CEESTEM, vol. 1, núm. 3, Centro de Estudios Económicos y Sociales del Tercer Mundo, 1981.

Gutman, P., "Medio ambiente urbano, interrogantes y reflexiones" en La planificación ambiental en Venezuela, Documentos del proyecto Macrosistemas Ambientales Venezolanos, Serie de Publicaciones núm. 2, Caracas, 1980.

Habermas, J., "Técnica y ciencia como ideologia", Revista de Ciencias Sociales y Humanidades, Universidad Autónoma Metropolitana, Azcapotzalco, vol. II, núm. 3, 1981.

JARAMILlo, S. y SCHTEINGART, M., "Acumulación de capital y producción de vivienda en América Latina: Estudio de casos", por aparecer en J. Walton (comp.), Capital and Labor in the Urbanized World, Londres, Sage, 1983. 
KADE, G., "The economics of pollution and the interdisciplinary approach to environmental planning" (Introducción), International Social Science Journal, vol. XXII, 1970.

KAPP, K. W., "Ruptura y protección del medio ambiente", Socialismo y medio ambiente, Barcelona, Colección Punto y Línea, Gustavo Gili, 1976.

LEFF, E., "Sobre la articulación de las ciencias en la relación naturaleza-sociedad" en E. Leff (comp.) Biosociologia y articulación de las ciencias, Universidad Nacional Autónoma de México, 1981.

Lipietz, A., Le tribut foncier urbain, París, Maspero, 1974.

LoJKINE, J., El marxismo, el Estado y la cuestión urbana, Méxiço, Siglo XXI, 1978.

Margalef, R., Ecologia, Barcelona, Planeta, 1981.

MURTHY, K. K., "Energy conservation: the global imperative", Impact of Science on Society, Unesco, vol. 28, núm. 2, 1978.

Odum, E., Ecologia, México, Compañía Continental, 1977.

RoFmAN, A., "El rol de la ciudad como síntesis de procesos sociales y procesos naturales", en La planificación ambiental en Venezuela, op. cit.

Sánchez, V., Giza, B., Legros, M. y Licona, A., Glosario de términos sobre medio ambiente, Documento de trabajo, El Colegio de México, 1980.

SAGASTI, Francisco R., El factor tecnológico en la teoria del desarrollo económico, Jornadas 94, El Colegio de México, 1981.

Sánchez, V., Giza, B., Legros, M. y Licona, A., "Problemas ambientales de América Latina", en Szekely F. (comp.), El medio ambiente en México y América Latina, México, Nueva Imagen, 1978.

SCHTEINGART, M., "El sector inmobiliario capitalista y las formas de apropiación del suelo urbano: El caso de México", Demografia y Economia, vol. XIII, núm. 4(40), 1979.

SEJENOVICH, H., "La concepción ambiental en el análisis de los asentamientos humanos: el caso de México", Lecturas del CEESTEM, vol. 1, núm. 3, Centro de Estudios Económicos y Sociales del Tercer Mundo, 1981.

Toledo, V., "Intercambio ecológico e intercambio en el proceso productivo primario", en E. Leff, op. cit.

Topalov, $\mathrm{CH}$., Capital et propiété fonciere, París, Centre de Sociologie Urbaine, 1972.

——_ La Urbanización capitalista, México, Edicol, 1972. 